\title{
Random cubic planar graphs revisited
}

\author{
Marc Noy \\ Departament de Matemàtiques, Universitat Politècnica de Catalunya \\ Barcelona Graduate School of Mathematics, Spain \\ Clément Requilé \\ Institut für Mathematik, Freie Universität Berlin \\ Berlin Mathematical School, Germany \\ Juanjo Rué \\ Departament de Matemàtiques, Universitat Politècnica de Catalunya \\ Barcelona Graduate School of Mathematics, Spain
}

\begin{abstract}
The goal of our work is to analyze random cubic planar graphs according to the uniform distribution. More precisely, let $\mathcal{G}$ be the class of labelled cubic planar graphs and let $g_{n}$ be the number of graphs with $n$ vertices. Then each graph in $\mathcal{G}$ with $n$ vertices has the same probability $1 / g_{n}$. This model was analyzed first by Bodirsky et al. [1], and here we revisit and extend their work. The motivation for this revision is twofold. First, some proofs in [1] where incomplete with respect to the singularity analysis and we aim at providing full proofs. Secondly, we obtain new results that considerably strengthen those in [1] and shed more light on the structure of random cubic planar graphs. We present a selection of our results on asymptotic enumeration and on limit laws for parameters of random graphs.
\end{abstract}

Keywords: random planar graphs, analytic combinatorics, asymptotic enumeration, limit laws 


\section{Results on enumeration}

Theorem 1.1 The number $c_{n}$ of connected cubic planar graphs with $n$ vertices is asymptotically

$$
c_{n} \sim c \cdot n^{-7 / 2} \gamma^{n} n !,
$$

with $c \approx 0.030487$ and $\gamma=\rho^{-1} \approx 3.132591$, where $\rho \approx 0.319523$ is the smallest positive root of the equation

$729 x^{12}+17496 x^{10}+148716 x^{8}+513216 x^{6}-7293760 x^{4}+279936 x^{2}+46656=0$,

Theorem 1.2 The number $g_{n}$ of cubic planar graphs with $n$ vertices is asymptotically

$$
g_{n} \sim g \cdot n^{-7 / 2} \gamma^{n} n !
$$

where $\gamma$ is as in Theorem 1.1 and $g \approx 0.030505$. As a consequence, the limiting probability $p$ that a random cubic planar graph is connected is equal to

$$
p=\frac{c}{g} \approx 0.999397 \text {. }
$$

The previous theorems were stated in [1, Theorem 2] in a less precise way and with incomplete proofs regarding the singularity analysis. Our first goal is to provide a full proof of these estimates. We remark that the actual value of $p$ was not computed in [1]. As we will see later, $p$ can be computed exactly using the so-called dissymmetry theorem for tree-like structures. We also remark that some of the constants given in [1] are slightly incorrect.

Theorem 1.3 The number $h_{n}$ of cubic planar multigraphs is asymptotically

$$
h_{n} \sim h \cdot n^{-7 / 2} \gamma_{m}^{n} n !
$$

with $h \approx 0.115965$ and $\gamma_{m}=\rho_{m}^{-1} \approx 3.985537$, where $\rho_{m} \approx 0.250907$ is the smallest positive root of

$729 x^{12}-17496 x^{10}+148716 x^{8}-513216 x^{6}-7293760 x^{4}-279936 x^{2}+46656=0$.

The same estimate holds for the number of connected cubic planar multigraphs, but with $h$ replaced by the constant $h^{\prime} \approx 0.104705$. The limiting probability of connectivity is

$$
p_{m}=\frac{h^{\prime}}{h} \approx 0.902905 .
$$

This result is also claimed in [3] without a detailed proof, but the equation defining $\rho_{m}$ is incorrect, as well as the claimed value $\gamma_{m} \approx 3.973$. 
We remark that the proof needs again an application of the dissymmetry theorem, since the presence of loops and multiple edges does not allow us, as for simple graphs, to relate directly the number of graphs rooted at a vertex with those rooted at an edge.

\section{Results on limit laws}

Our main results in this section deal with the number of copies of a fixed subgraph. We start with the number of triangles.

Theorem 2.1 Let $X_{n}$ be the number of triangles in a random cubic planar graph. Then $X_{n}$ is asymptotically normal with moments

$$
\mathbf{E} X_{n} \sim \mu n, \quad \operatorname{Var} X_{n} \sim \lambda n,
$$

where

$$
\mu \approx 0.121974, \quad \lambda \approx 0.064985 .
$$

It was proved in [1] that $X_{n}$ is linear with high probability. Our result is a considerable sharpening of this fact. We wish to remark that this is the first time one is able to determine precisely the number of copies of a fixed graph $H$ containing a cycle in classes of random planar graphs. The proof, based on the so-called quasi-powers theorem, is technically involved and we are not able to extend it, for instance, to the number of quadrilaterals.

Using the combinatorial decompositions introduced for counting triangles we can enumerate triangle-free graphs.

Theorem 2.2 The number $t_{n}$ of triangle-free cubic planar graphs with $n$ vertices is asymptotically

$$
t_{n} \sim t \cdot n^{-7 / 2} \gamma_{t}^{n} n !
$$

where $\gamma_{t} \approx 2.646686$ and $t>0$.

Our next results concern the number of copies of graphs which are close to being cubic. We define a cherry as a planar graph in which all vertices have degree 3 except for one distinguished vertex of degree 1 . The smallest cherry has 6 vertices and is obtained by subdividing an edge of $K_{4}$ and adding one vertex of degree one.

Theorem 2.3 Let $X_{H, n}$ be the number of copies of a fixed cherry $H$ in a random cubic planar graph. Then $X_{H, n}$ is asymptotically normal with moments

$$
\mathrm{E} X_{H, n} \sim \mu n, \quad \operatorname{Var} X_{H, n} \sim \eta n,
$$


where $\mu$ and $\eta$ depend on the constant $\gamma$ in Theorem 1.1 and $H$.

The previous result improves on [1], where it is shown that the number of copies of a fixed cherry is linear w.h.p.

We comment briefly on the notable differences of this model with the classical model of random cubic (not necessarily planar) graphs [7]. A random cubic graph is w.h.p. 3-connected. Also, the number of triangles follows a Poisson law with expectation $4 / 3$. Finally a random cubic planar graph is 3-colorable, whereas a random cubic graph has a (small) probability of containing $K_{4}$ as a component, hence of being 4-chromatic.

\section{Discussion of the proofs}

All graphs are labelled and the corresponding generating functions are exponential, expect for the family of rooted triangulations.

The starting point is, as in [1], the enumeration of cubic 3-connected planar graphs, which by duality are in bijection with planar triangulations. Tutte showed in one of his seminal papers on the enumeration of maps [6] the (ordinary) generating function of rooted 3-connected triangulations is given by

$$
T(z)=U(z)(1-2 U(z)), \quad \text { where } z=U(1-U)^{3} .
$$

The unique singularity of $T$ is $\tau=27 / 256$, and we have $U(\tau)=1 / 4, T(\tau)=$ $1 / 8$. If now $M(x, y)$ is the GF for labelled 3-connected cubic planar graphs rooted at a directed edge, where $x$ marks vertices and $y$ marks edges, by duality we have

$$
M(x, y)=\frac{1}{2}\left(T\left(x^{2} y^{3}\right)-x^{2} y^{3}\right) .
$$

We define networks as in [1], but deviate slightly from the notation there. A network is a connected cubic multigraph $G$ with an ordered pair of adjacent vertices $(s, t)$, such that the graph obtained by removing the edge $s t$ is simple. We show that the generating function $D(x)$ satisfies

$$
(1+D) \sqrt{\left(2+x^{2}\right)^{2}-4 x^{2} D}-2-T\left(x^{2}(1+D)^{3}\right)=0 .
$$

Finally, we let $C(x)$ be the generating function of connected cubic planar graphs. By double counting the edges, one shows that

$$
3 x C^{\prime}(x)=\frac{L(x)}{x^{2}}+D(x)-L(x)-x^{2} D(x)-L(x)^{2},
$$


where $L(x)$ is the generating function of loop networks, given by

$$
L(x)=\frac{1}{2}\left(2+x^{2}-\sqrt{\left(2+x^{2}\right)^{2}-4 x^{2} D(x)}\right) .
$$

When solving (2) there are two possible sources of singularities for $D$ : either a singularity coming from $T$ or a branch point. We show that there is no branch point (this is a key point not addressed in [1]), hence the singularity $\rho$ comes from $T$. By eliminating from the previous equations, we show that $\rho$ is as claimed in Theorem 1.1.

Next we compute the singular expansion of $D(x)$ at $\rho$, which is of the form

$$
D(x)=D_{0}+D_{2} X^{2}+D_{3} X^{3}+O\left(X^{4}\right), \quad X=\sqrt{1-x / \rho} .
$$

The coefficients $D_{i}$ are computed from (2) and the known expansion

$$
T(z)=\frac{1}{8}-\frac{3}{16} Z^{2}+\frac{\sqrt{6}}{24} Z^{3}+O\left(Z^{4}\right), \quad Z=\sqrt{1-z / \tau} .
$$

Finally, plugging the singular expansion of $D$ into Equation (3) and integrating we obtain an expansion for $C(x)$. By singularity analysis (see [4]), the estimate in Theorem 1.1 follows.

In order to prove Theorem 1.2, we proceed as follows. The limiting probability of a random graph being connected is (see [5]) $p=e^{-C(\rho)}$. Hence, with the notations of theorems 1.1 and 1.2 , we have $c_{n} \sim p \cdot g_{n}$. In order to compute $C(\rho)$ we need an explicit expression for $C(x)$. The natural approach is to integrate (3), but this needs integrating $D(x)$, and we are not able to do it algebraically. Instead we compute $C(x)$ directly by using the dissymmetry theorem for tree-decomposable structures, as in [2]. In other words, we integrate combinatorially, instead of algebraically. We obtain an explicit (too long to reproduce here) expression for $C(x)$ in terms of $D(x)$, from which we can compute $C(\rho)$.

The proof of the Gaussian limit law is based on the quasi-powers theorem [4]. In the case of Theorem 2.1, we add a variable $u$ for marking triangles and we show that the associated bivariate generating function $D(x, u)$ of networks has a unique dominant singularity $\rho(u)$ and locally an expansion of the form

$$
D(x, u)=D_{0}(u)+D_{2}(u) X^{2}+D_{3}(u) X^{3}+O\left(X^{4}\right), \quad X=\sqrt{1-x / \rho(u)} .
$$

The probability generating function $p_{n}(u)$ of the random variable counting 
triangles is

$$
p_{n}(u)=\frac{\left[x^{n}\right] D(x, u)}{\left[x^{n}\right] D(x, 1)} .
$$

By singularity analysis we have an estimate near $u=1$ (uniform on $u$ ) of the form

$$
p_{n}(u)=A(u) B(u)^{n}\left(1+O\left(n^{-1}\right)\right) .
$$

Thus $p_{n}(u)$ is 'close' to being an exact power and we deduce a central limit theorem for the number of triangles. From the values $p_{n}(1)$ and $p_{n}^{\prime}(1)$ one gets an estimate for the first and second moments. The main difficulty here is to keep track of the triangles in the combinatorial decompositions and to locate the singularities. In fact, the equations defining $D(x, u)$ are considerably involved. Once this is done, it translates easily into connected and arbitrary cubic planar graphs.

Theorem 2.2 is obtained setting $u=0$ in $D(x, u)$. Since 0 is away from 1 , care must be taken to guarantee that, once more, there is no branch point and the singularity comes from that of triangulations.

\section{References}

[1] M. Bodirsky, M. Kang, M. Löffler, C. McDiarmid. Random cubic planar graphs. Random Structures \&3 Algorithms 30 (2007), 78-94.

[2] G. Chapuy, É. Fusy, M. Kang, B. Shoilekova. A complete grammar for decomposing a family of graphs into 3-connected components. Electron. J. Combin. 15 (2008), no. 1, Research Paper 148, 39 pp.

[3] W. Fang, M. Kang, M. Moßhammer, P. Sprüssel. Cubic graphs and related triangulations on orientable surfaces (2016) arXiv:1603.01440.

[4] P. Flajolet, R. Sedgewick. Analytic Combinatorics. Cambridge University Press, Cambridge, 2009.

[5] O. Giménez, M. Noy. Asymptotic enumeration and limit laws of planar graphs. J. Amer. Math. Soc. 22 (2009), 309-329.

[6] W. T. Tutte. A census of planar triangulations. Canad. J. Math. 14 (1962) $21-38$.

[7] N. C. Wormald, Models of random regular graphs, in Surveys in Combinatorics, 1999, pp. 239-298, Cambridge University Press, Cambridge (1999). 